\title{
Distance-restricted matching extension in triangulations of the torus and the Klein bottle
}

\author{
R. E. L. Aldred \\ Department of Mathematics and Statistics \\ University of Otago \\ P.O. Box 56, Dunedin, New Zealand \\ raldred@maths.otago.ac.nz
}

\author{
Jun Fujisawa* \\ Faculty of Business and Commerce \\ Keio University \\ Yokohama, 223-8521 Japan \\ fujisawa@fbc.keio.ac.jp
}

Submitted: Dec 7, 2012; Accepted: Aug 4, 2014; Published: Sep 18, 2014

Mathematics Subject Classifications: 05C70, 05C10

\begin{abstract}
A graph $G$ with at least $2 m+2$ edges is said to be distance $d m$-extendable if for any matching $M$ in $G$ with $m$ edges in which the edges lie pair-wise distance at least $d$, there exists a perfect matching in $G$ containing $M$. In a previous paper, Aldred and Plummer proved that every 5-connected triangulation of the plane or the projective plane of even order is distance $5 m$-extendable for any $m$. In this paper we prove that the same conclusion holds for every triangulation of the torus or the Klein bottle.
\end{abstract}

Keywords: distance restricted matching extension; triangulation; toroidal graph; Klein bottle graph; non-contractible cycle; separating cycle

\section{Introduction}

We consider only simple graphs, that is, without loops or multiple edges. A set $M$ of edges in a graph is said to be a matching if no two members of $M$ share a vertex. $A$ perfect matching in a graph $G$ is a matching in $G$ which covers all the vertices of $G$. If a matching $M$ in $G$ is a subset of a perfect matching in $G$, then $M$ is said to be extendable in $G$, and a graph with at least $2 m+2$ vertices in which every matching of size $m$ is extendable is called $m$-extendable.

A closed curve on a closed surface $F^{2}$ is a continuous function $\ell: S^{1} \rightarrow F^{2}$ or its image, where $S^{1}$ is the 1 -dimensional sphere, that is, $\left\{(x, y) \in \boldsymbol{R}^{2} \mid x^{2}+y^{2}=1\right\}$. A

\footnotetext{
*This work was supported by JSPS Grant-in-Aid for Young Scientists (B).
} 
closed curve $\ell$ is called simple if the function $\ell$ is an injection, that is, the curve does not cross itself. Moreover, if a simple closed curve $\ell$ bounds a 2 -cell on $F^{2}$, then $\ell$ is called contractible, and otherwise $\ell$ is called non-contractible.

In this paper we deal with a graph $G$ embedded on some closed surface, and investigate when a matching $M$ is extendable. For a matching with two edges, Plummer [10] proved the following.

Theorem 1. Every 5-connected planar graph of even order is 2-extendable.

To show a similar result for any locally planar graph $G$ embedded in a surface $F^{2}$, Kawarabayashi et al. added a condition on the representativity $\rho(G)$ (the minimum number $r$ such that any non-contractible simple closed curve on $F^{2}$ meets $G$ in at least $r$ places), and proved the following.

Theorem 2 ([7]). Every 5-connected graph $G$ of even order embedded on a closed surface $F^{2}$, except the sphere, is 2-extendable if $\rho(G) \geqslant 7-2 \chi\left(F^{2}\right)$, where $\chi\left(F^{2}\right)$ is the Euler characteristic of $F^{2}$.

Moreover, they proved that there are infinitely many 5-connected triangulations $G$ of $F^{2}$ with $\rho(G)=3$ that are not 2-extendable, for any closed surface $F^{2}$ except the sphere and the projective plane. Therefore, the condition on the representativity is necessary in Theorem 2 when $F^{2}$ is not the projective plane.

It is known that no planar graph with at least 8 vertices is 3 -extendable $([9])$. Later, Aldred and Plummer ([1], [3], [4]) studied the relationship between the distance between a set of edges and their extendability. A graph $G$ with at least $2 m+2$ edges is said to be distance $d m$-extendable if any matching $M$ in $G$ with $m$ edges in which the edges lie pair-wise distance at least $d$ is extendable. In particular, several important results for proximity based matching extension have been obtained for triangulations, i.e. simple graphs which are embedded on surfaces so that each face is bounded by a triangle.

Theorem 3 ([1]). Every 5-connected triangulation of the plane of even order is distance 2 3-extendable.

It follows from Theorem 3 that, in any 5-connected triangulation of the plane of even order, any non-extendable matching with three edges contains a pair of edges at distance 1.

It is shown in [3] that the conclusion of Theorem 3 cannot be extended to "distance 2 4-extendable". However, if the pair-wise distance of the given matching is increased, then we can extend 4 or more edges.

Theorem 4 ([3], [4]). Every 5-connected triangulation of the plane of even order is distance 3 4-extendable. Moreover, there exist infinitely many 5-connected triangulations of the plane of even order which are not distance 3 10-extendable.

Theorem 5 ([4]). Every 5-connected triangulation of the plane of even order is distance 4 7-extendable. 
Note that, in the distance 2 or 3 case, there exists a maximum value for the number of edges which may be extended. Though we do not know whether such a maximum exists in the distance 4 case, it disappears in distance 5 case.

Theorem 6 ([4]). Every 5-connected triangulation of the plane of even order is distance 5 m-extendable for any $m$.

It is also shown in [4] that Theorem 6 still holds when "plane" is replaced by "projective plane". For graphs on the torus or Klein bottle, it follows from Mizukai et al.'s result [8] that every 5-connected triangulation of the torus or the Klein bottle of even order is distance 32 -extendable for any $m$. This result is sharp in the following sense.

Proposition 7 (Aldred and Plummer, [5]). There are 5-connected triangulations of both the torus and the Klein bottle of even order which are not distance 3 3-extendable.

The main purpose of this paper is to extend Theorem 6 to graphs on the torus and the Klein bottle. In the above results, we always find a difference between the planar case and the toroidal or the Klein bottle cases: when we extend Theorem 1 to Theorem 2, the additional condition on representativity is necessary, and in Proposition 7, the number of the edges which may be extended is reduced compared to the planar case. In contrast to these situations, we can extend Theorem 6 without any extra condition.

Theorem 8. Let $G$ be a 5-connected triangulation of the torus or the Klein bottle of even order. If $m \geqslant 0$ and $G$ has at least $2 m+2$ vertices, then $G$ is distance $5 m$-extendable.

It is shown in [5] that there exist 4-connected triangulations of both the torus and the Klein bottle of even order which are not 1-extendable, and so the connectivity condition in Theorem 8 cannot be relaxed. Next we show that the "triangulation" hypothesis cannot be dropped in Theorem 8. Let $G_{0}$ and $H_{0}$ be the graphs shown in Figures 1 and 2, respectively.

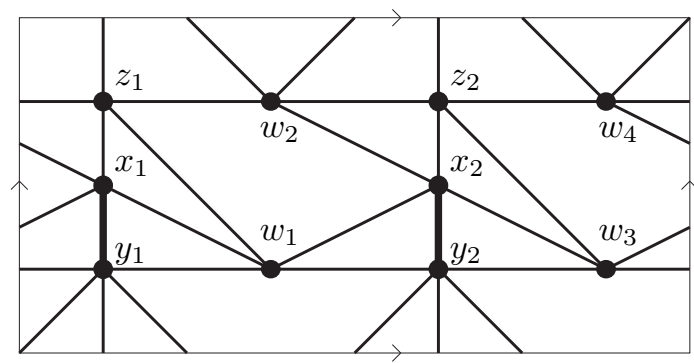

Figure 1: the graph $G_{0}$.

Let $G$ be the graph obtained from $G_{0}-\left\{w_{1}, w_{2}, w_{3}, w_{4}\right\}$ by adding 4 copies $H_{1}, H_{2}$, $H_{3}, H_{4}$ of $H_{0}-\{v\}$ and joining the five vertices of degree 4 in $H_{i}$ and the five neighbors of $w_{i}$ in $G_{0}$ by a perfect matching for $i=1,2,3,4$ so that the resulting graph is toroidal (note that such a matching is unique up to symmetry). Then, since both $G_{0}$ and $H_{0}$ are 


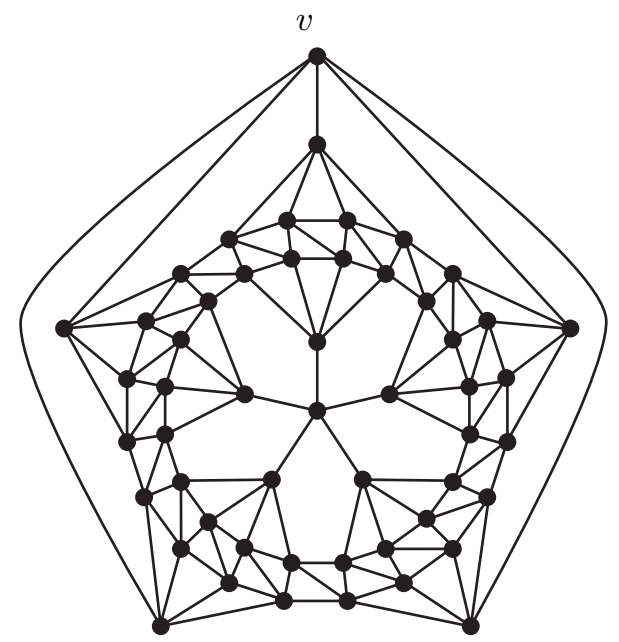

Figure 2: the graph $H_{0}$.

5-connected, $G$ is also 5-connected. Moreover, since $G-\left\{x_{1}, y_{1}, z_{1}, x_{2}, y_{2}, z_{2}\right\}$ has 4 odd components, $\left\{x_{1} y_{1}, x_{2} y_{2}\right\}$ is not extendable in $G$. Thus $G$ is not distance 52 -extendable, though $G$ is a 5 -connected toroidal graph of even order. Note that we obtain larger examples by recursively exchanging a vertex in $V(G) \backslash\left\{x_{1}, y_{1}, z_{1}, x_{2}, y_{2}, z_{2}\right\}$ for the graph $H_{0}-\{v\}$ and adding 5 edges as indicated in the above. Moreover, notice that $G$ can be embedded on the Klein bottle (See Figure 3).

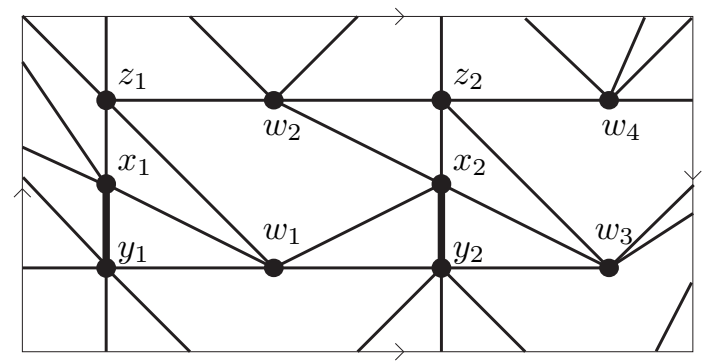

Figure 3: the graph $G_{0}^{\prime}$.

Though Theorem 8 is best possible, it remains to be studied whether we can replace "distance 5" with "distance 4" in Theorem 8 or not.

We prove Theorem 8 in the next section. In the rest of the present section, we introduce terminology and notation used in the proof. Two closed curves $\ell_{1}$ and $\ell_{2}$ on a closed surface $F^{2}$ are said to be homotopic to each other on $F^{2}$ if there exists a continuous function $\Phi:[0,1] \times S^{1} \rightarrow F^{2}$ such that $\Phi(0, x)=\ell_{1}(x)$ and $\Phi(1, x)=\ell_{2}(x)$ for each $x \in S^{1}$.

A cycle $C$ of a graph $G$ on $F^{2}$ is called contractible (resp. non-contractible) if the edges 
of $C$ induce a contractible (resp. non-contractible) curve on $F^{2}$. A non-contractible cycle $C$ of a graph $G$ on $F^{2}$ is called separating if the curve on $F^{2}$ induced by the the edges of $C$ separates $F^{2}$. Note that a separating cycle does not necessarily separate the graph, and so $G-V(C)$ may be connected. In the graph $G_{0}^{\prime}$ in Figure $3, z_{1} w_{2} z_{2} w_{4} y_{1} w_{1} y_{2} w_{3} x_{1} z_{1}$ is a separating cycle. By definition, any non-contractible cycle of the graph on the torus is non-separating.

A closed curve $\ell$ on $F^{2}$ is said to be 1-sided if the tubular neighborhood of $\ell$ forms a Möbius band, and 2-sided otherwise. Moreover, a cycle $C$ of a graph $G$ on $F^{2}$ is called 1-sided (resp. 2-sided) if the edges of $C$ induce a 1-sided (resp. 2-sided) curve on $F^{2}$. In the graph $G_{0}^{\prime}$ in Figure $3, z_{1} w_{2} z_{2} w_{4} z_{1}$ is a 1-sided cycle, and $z_{1} w_{2} z_{2} w_{4} y_{1} w_{1} y_{2} w_{3} x_{1} z_{1}$ is a 2 -sided cycle. By definition, any cycle of the graph on the torus is 2-sided.

Let $C$ be a 2-sided cycle of a graph $G$ on $F^{2}$ and let $D_{1}, D_{2}$ be vertex-disjoint subgraphs of $G-V(C)$. If there exist two edges $x_{1} y_{1}$ and $x_{2} y_{2}$ such that $x_{1}, x_{2} \in V(C), y_{i} \in V\left(D_{i}\right)$ for $i=1,2$ and $x_{1} y_{1}$ is adjacent to $x_{1}$ on the right-hand side of $C$ and $x_{2} y_{2}$ is adjacent to $x_{2}$ on the left-hand side of $C$ for an arbitrary orientation of $C$, then we say that $D_{1}$ and $D_{2}$ are adjacent to $C$ on opposite sides of $C$.

Let $e$ be an edge and $F$ be a set of edges. The set of two vertices which are incident with $e$ is denoted by $V(e)$, and we denote $V(F)=\bigcup_{e \in F} V(e)$. Moreover, the length of a shortest path joining a vertex $v$ and $V(e)$ is denoted by $d(v, e)$. For other terminology and notation, we refer the reader to $[6]$.

\section{Proof of Theorem 8}

Suppose that $m$ is the smallest integer for which distance $5 \mathrm{~m}$-extendable fails to hold on the torus (resp. the Klein bottle). Since every 5-connected triangulation of the torus (resp. the Klein bottle) of even order is 1-extendable by Theorem 4.3 (b) (resp. Theorem $5.3(\mathrm{~b}))$ of [2], we have $m \geqslant 2$. Let $M=\left\{e_{1}, \ldots, e_{m}\right\}$ be a set of $m$ edges at mutual distance 5 or more which does not extend to a perfect matching. Then since $G^{\prime}=G-V(M)$ does not have a perfect matching, by Tutte's theorem there is a barrier set $S \subseteq V(G)$ such that $c_{o}\left(G^{\prime}-S\right) \geqslant|S|+2$, where $c_{o}(H)$ denotes the number of odd components of a graph $H$. By the minimality of $m$ we have $c_{o}\left(G^{\prime}-S\right)=|S|+2$. Now we choose such an $S$ to be as small as possible.

Let $K=S \cup V(M)$. Assume that there exists an odd component $D$ of $G-K$ and two vertices $r_{1}$ and $r_{2}$ in $S$ which lie in different components of $G-V(D)$. Let $H^{\prime}$ be the component of $G-V(D)$ containing $r_{1}$, let $R_{1}=V\left(H^{\prime}\right) \cap S$ and let $R_{2}=S \backslash R_{1}$. Then $c_{o}\left(G-R_{i}\right) \geqslant\left|R_{i}\right|+2$ holds for $i=1$ or 2 , which contradicts the minimality of $S$. Therefore,

$$
G-V(D) \text { is connected for every component } D \text { of } G-K \text {. }
$$

We construct the bipartite distillation $G^{*}$ of graph $G$ as in the proof of Theorem 2.1 of [4]. That is, delete all the even components of $G-K$ and all edges within $G[K]$ and next contract each of the odd components of $G-K$ to a single vertex and delete any loops 
and multiple edges. We call the resulting bipartite graph $G^{*}$. Since $G^{*}$ is a minor of $G$, we can embed $G^{*}$ on the same closed surface as $G$, the torus or the Klein bottle. Hence it follows from Euler's formula that $\left|E\left(G^{*}\right)\right| \leqslant 2\left|V\left(G^{*}\right)\right|=2(2|S|+2+2 m)=4|S|+4 m+4$. On the other hand, since $G$ is 5-connected, every odd component of $G-K$ has at least five vertices of attachment in $K$. And so $\left|E\left(G^{*}\right)\right| \geqslant 5(|S|+2)$. Thus

$$
|S| \leqslant 4 m-6 \text {. }
$$

Suppose a vertex $s \in S$ has neighbors in at most two components of $G^{\prime}-S$. Let $S^{\prime}=S-\{s\}$, then $G^{\prime}-S^{\prime}$ has at least $|S|+1=\left|S^{\prime}\right|+2$ odd components, which contradicts the minimality of $S$. Therefore each vertex $s \in S$ has neighbors in at least three components of $G^{\prime}-S$. Take $s \in S$ and let the neighbors of $s$ be $v_{1}, v_{2}, \ldots, v_{l}$ such that $s v_{i} v_{i+1}$ is a face of $G$ for each $i$ with $1 \leqslant i \leqslant l-1$. Let $D_{1}, D_{2}$ and $D_{3}$ be three odd components of $G^{\prime}-S$ containing a neighbor of $s$. Without loss of generality, we may assume that $v_{1} \in D_{1}, v_{p} \in D_{2}$ and $v_{q} \in D_{3}$ with $1<p<q$. Then, since each $D_{i}$ is a different odd component of $G-K$ and $G$ is a triangulation, we have three vertices $v_{p^{\prime}}, v_{q^{\prime}}, v_{r^{\prime}} \in K$ such that $1<p^{\prime}<p<q^{\prime}<q<r^{\prime}$. From this observation, it follows that each vertex $s$ in $S$ has at least three distinct neighbors $t_{1}, t_{2}$ and $t_{3}$ in $K$.

For each $e_{i} \in M$, let $S\left(e_{i}\right)=\left\{s \in K \mid 1 \leqslant d\left(s, e_{i}\right) \leqslant 2\right\}$. Since edges in $M$ are at least distance 5 apart, it holds that $S\left(e_{i}\right) \subseteq S$ and $S\left(e_{i}\right) \cap S\left(e_{j}\right)=\emptyset$ for $e_{i}, e_{j} \in M$ with $i \neq j$. The next claim plays a central role in our proof of Theorem 8 .

Claim 1. For each edge $e_{i}=x y \in M$, at least one of the following holds.

(i) $\left|S\left(e_{i}\right)\right| \geqslant 4$.

(ii) $\left|S\left(e_{i}\right)\right| \geqslant 2$ and $G\left[S\left(e_{i}\right) \cup\{x, y\}\right]$ contains two non-homotopic non-contractible cycles of length at most 4 .

(ii') $\left|S\left(e_{i}\right)\right| \geqslant 2$ and $G\left[S\left(e_{i}\right) \cup\{x, y\}\right]$ contains a separating 2 -sided non-contractible cycle of length at most 4.

(ii") $\left|S\left(e_{i}\right)\right| \geqslant 2$ and $G\left[S\left(e_{i}\right) \cup\{x, y\}\right]$ contains a 1-sided non-contractible cycle of length at most 4 .

(iii) $\left|S\left(e_{i}\right)\right| \geqslant 3, G\left[S\left(e_{i}\right) \cup\{x, y\}\right]$ contains a non-separating 2-sided non-contractible cycle of length at most 4 and there exists a component of $G-K$ which has a neighbor of a vertex of $S\left(e_{i}\right)$ and contains a non-separating 2 -sided non-contractible cycle.

Note that (ii') or (ii") of the above claim apply only to the case $G$ is embedded in the Klein bottle.

Proof. We assume that none of (i), (ii), (ii') or (ii") holds, and prove (iii).

Note that $e_{i}$ has neighbors in at least two odd components of $G-K$, as otherwise $c_{o}\left(G^{\prime \prime}-S\right)=|S|+2$, where $G^{\prime \prime}=G-(V(M) \backslash\{x, y\})$, contradicting the minimality of $m$. Now contract $e_{i}$ to a single vertex $w_{i}$ and delete the created loop. Since $G$ is a 5 -connected 


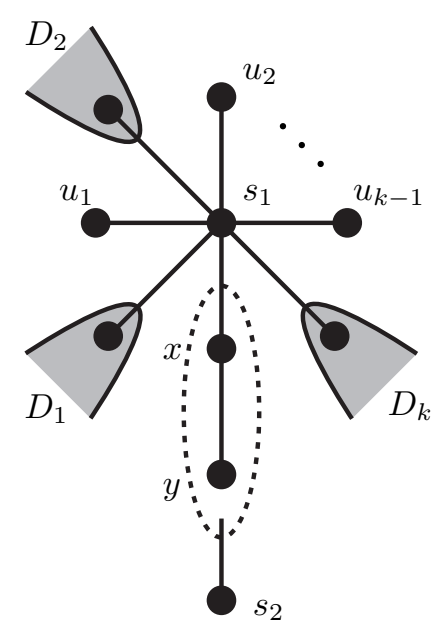

Figure 4: Vertices around $s_{1}$

triangulation, this contraction creates exactly two pairs of multiple edges which bound a 2-cell (in particular, such pairs do not share an edge, and each pair induces a digon). Here delete one of the edges of each pair, and let $G_{i}$ be the resulting graph. Then $G_{i}$ is a triangulation as well. Let the neighbors of $w_{i}$ in $G_{i}$ be $v_{1}, v_{2}, \ldots, v_{l}$ such that $w_{i} v_{j} v_{j+1}$ is a face of $G_{i}$ for each $j$ with $1 \leqslant j \leqslant l-1$. Without loss of generality, we may assume that $v_{1}$ and $v_{p}$ belong to different odd components of $G-K$ for some $p$. Let $D$ (resp. $D^{\prime}$ ) be the component of $G-K$ which contains $v_{1}$ (resp. $v_{p}$ ). Then we have two vertices $v_{p^{\prime}}, v_{p^{\prime \prime}} \in S$ such that $1<p^{\prime}<p<p^{\prime \prime} \leqslant l$. Let $s_{1}=v_{p^{\prime}}$ and $s_{2}=v_{p^{\prime \prime}}$.

Now $s_{1}$ is a neighbor of the edge $e_{i}=x y$ in $G$. Without loss of generality, we may assume that $s_{1} x \in E(G)$. As we noted before, $s_{1}$ has neighbors in at least three odd components and has at least three neighbors in $K$. Let $D_{1}, D_{2}, \ldots, D_{k}$ be such components $(k \geqslant 3)$, and let $x, u_{1}, u_{2}, \ldots, u_{k-1}$ be such neighbors, where $k \geqslant 3$ and $x, N\left(s_{1}\right) \cap D_{1}, u_{1}$, $N\left(s_{1}\right) \cap D_{2}, u_{2}, N\left(s_{1}\right) \cap D_{3}, \ldots u_{k-1}, N\left(s_{1}\right) \cap D_{k}$ appear in this order when we traverse $N\left(s_{1}\right)$ in the clockwise direction (see Figure 4).

Subclaim 1. $G\left[S\left(e_{i}\right) \cup\{x, y\}\right]$ contains a non-contractible cycle $C$ of length at most 4 which contains the edge $s_{1} x$. Moreover, if $C$ is a 2-sided cycle, then there exist two distinct components of $G-K$ which are adjacent to $C$ on opposite sides of $C$.

Proof. In the case $s_{1}=s_{2}$, let $C=C=x y s_{1} x$. By the construction of $G_{i}, C$ is a non-contractible cycle. Moreover, if $C$ is a 2-sided cycle, then $D$ and $D^{\prime}$ are adjacent to $C$ on opposite sides of $C$. Thus $C$ is a desired cycle.

In the case $u_{j}=y$ for some $j$, let $C=s_{1} u_{j} x s_{1}$. If $C$ is a contractible cycle, then $D_{1}$ and $D_{k}$ are contained in different components of $G-V(C)$, contradicting the 5-connectedness of $G$. Therefore $C$ is a non-contractible cycle. If, in addition, $C$ is a 2 -sided cycle, then $D_{1}$ and $D_{k}$ are adjacent to $C$ on opposite sides of $C$, and thus $C$ is a desired cycle. 
Therefore, we may assume that $s_{1} \neq s_{2}$ and $u_{j} \neq y$ for each $j$. Since (i) does not hold, it follows that $u_{r}=s_{2}$ for some $r$. Then either $s_{1} u_{r} y x s_{1}$ or $s_{1} u_{r} x s_{1}$ is a cycle, say $C$. If $C$ is a contractible cycle, then $D_{1}$ and $D_{k}$ are contained in different components of $G-V(C)$, contradicting the 5-connectedness of $G$. Therefore $C$ is a non-contractible cycle. Moreover, if $C$ is a 2-sided cycle, then $D_{1}$ and $D_{k}$ are adjacent to $C$ on opposite sides of $C$, and thus $C$ is a desired cycle. This establishes Subclaim 1.

Assume $C$ is as in Subclaim 1. If $C$ is a 1-sided cycle or a separating 2-sided cycle, then since $\left\{s_{1}, u_{1}, u_{2}, \ldots, u_{k}\right\} \backslash\{x, y\} \subseteq S\left(e_{i}\right)$, we have $\left|S\left(e_{i}\right)\right| \geqslant 2$. This contradicts the assumption that neither (ii') nor (ii") holds, and thus we may assume that $C$ is a nonseparating 2-sided cycle. Recall that $s_{1}$ has neighbors in at least three odd components. Without loss of generality, we may assume that $s_{1}$ is adjacent to $D_{1}$ and $D_{2}$ on the lefthand side of $C$ and is adjacent to $D_{k}$ on the right-hand side of $C$. Let $S^{\prime}\left(e_{i}\right)$ be the set of the vertices in $S\left(e_{i}\right)$ which are in $V(C)$ or are adjacent to $C$ on the left-hand side. We call a component $D$ of $G-K$ non-contractible if $D$ contains a non-contractible cycle, and otherwise we call $D$ contractible.

Subclaim 2. $\left|S^{\prime}\left(e_{i}\right)\right| \geqslant 3$.

Proof. If $|V(C) \cap S| \geqslant 2$, then since $(V(C) \cap S) \cup\left\{u_{1}\right\}$ is a subset of $S^{\prime}\left(e_{i}\right)$, Subclaim 2 is satisfied.

Thus we may assume $|V(C) \cap S|=1$, which implies $s_{1}=s_{2}$ and $C=x y s_{1} x$. Let the neighbors of $s_{1}$ in $G$ be $a_{1}, a_{2}, \ldots, a_{l}$ such that $s_{1} a_{j} a_{j+1}$ is a face of $G$ for each $j$ with $1 \leqslant j \leqslant l-1$. Without loss of generality, we may assume that $x=a_{1}, u_{1}=a_{p}$ and $y=a_{q}$. If there exists $j$ with $1<j<q$ and $j \neq p$ such that $a_{j} \in S\left(e_{i}\right)$, then since $\left\{s_{1}, u_{1}, a_{j}\right\} \subseteq S^{\prime}\left(e_{i}\right)$, the assertion holds. Thus we may assume that

$$
a_{j} \notin S\left(e_{i}\right) \text { for every } j \text { with } 1<j<q \text { and } j \neq p \text {. }
$$

Since $C$ is a non-separating 2-sided cycle, it follows that either $D_{1}$ or $D_{2}$ is a contractible component. Assume that $D_{1}$ is a contractible component. Let $T_{1}$ be the set of vertices of $K$ which are adjacent to $D_{1}$, then by (1) $T_{1}$ induces a closed trail. Let $b_{1} b_{2} b_{3} \ldots$ be such a closed trail, where $b_{1}=s_{1}$ and $b_{2}=u_{1}$. Moreover, let $l$ be the smallest number such that $b_{l} \in V(C)$ and the edge $b_{l-1} b_{l}$ is adjacent to $C$ on the left-hand side. By (3), we have $b_{l}=x$ or $y$. Thus $b_{l-1} \in S\left(e_{i}\right) \backslash\left\{s_{1}\right\}$. If $b_{l-1}=u_{1}$, then $D_{1}$ and $D_{2}$ are contained in different components of $G-\left\{s_{1} b_{l-1} b_{l}\right\}$, a contradiction. Therefore we have $b_{l-1} \neq u_{1}$, and hence $\left\{s_{1}, u_{1}, b_{l-1}\right\}$ implies the assertion. By the same argument, we also obtain the assertion in the case where $D_{2}$ is a contractible component. This establishes Subclaim 2.

If $D_{k}$ is a non-contractible component, then since $C$ is a non-separating 2-sided cycle, the non-contractible cycle contained in $D_{k}$ is also a non-separating 2-sided cycle. Hence (iii) follows from Subclaims 1 and 2. Thus we assume that $D_{k}$ is a contractible component. Let $T_{k}$ be the set of vertices of $K$ which are adjacent to $D_{k}$. Since $G$ is 5 -connected, we have $\left|T_{k}\right| \geqslant 5$, which implies $T_{k} \backslash V(C) \neq \emptyset$. Since $G\left[T_{k}\right]$ is connected, there exists $s^{\prime} \in T_{k} \backslash V(C)$ such that $s^{\prime}$ is adjacent to $C$ on the right-hand side. Then $s^{\prime} \in S\left(e_{i}\right)$. If 
$s^{\prime} \notin S^{\prime}\left(e_{i}\right)$, then $S^{\prime}\left(e_{i}\right) \cup\left\{s^{\prime}\right\}$ implies (i), a contradiction. On the other hand, if $s^{\prime} \in S^{\prime}\left(e_{i}\right)$, then since $s^{\prime}$ is adjacent to $C$ on both sides, (ii) holds. This contradiction completes the proof of Claim 1.

Claim 2. If (ii') or (ii") of Claim 1 holds for some $e_{i}$, then there exists at most one edge in $M \backslash\left\{e_{i}\right\}$ which satisfies (ii') or (ii") and no edge in $M \backslash\left\{e_{i}\right\}$ satisfies (ii) or (iii).

Proof. Recall that $G$ is embedded on the Klein bottle when (ii') or (ii") holds for $e_{i}$. Assume first that $e_{i}$ satisfies (ii') and let $C$ be a separating non-contractible cycle of length at most 4 contained in $G\left[S\left(e_{i}\right) \cup\{x, y\}\right]$. By cutting the Klein bottle along $C$ (and deleting the vertices in $C$ ), we obtain two Möbius bands. Since $G$ is 5 -connected, one of them cannot contain any vertex. Moreover, since $S\left(e_{i}\right) \cap S\left(e_{j}\right)=\emptyset$ for every $j \neq i$, the other Möbius band can contain at most one edge in $M$ which satisfies (ii') or (ii") and it cannot contain any edge in $M$ which satisfies (ii) or (iii).

Next assume that no edge in $M$ satisfies (ii') and assume that $e_{i}$ satisfies (ii"). Let $C$ be the 1-sided non-contractible cycle of length at most 4 contained in $G\left[S\left(e_{i}\right) \cup\{x, y\}\right]$, then by cutting the Klein bottle along $C$, we obtain a Möbius band. Since $S\left(e_{i}\right) \cap S\left(e_{j}\right)=\emptyset$ for every $j \neq i$, the Möbius band can contain at most one edge in $M$ which satisfies (ii"), and it cannot contain any edge in $M$ which satisfies (ii) or (iii). This establishes Claim 2 .

By Claim 2, if (ii') or (ii") of Claim 1 holds for some $e_{i}$, then we can choose $e_{j} \neq e_{i}$ so that each edge in $M \backslash\left\{e_{i}, e_{j}\right\}$ satisfies (i) of Claim 1. Then $|S| \geqslant \sum_{i=1}^{m}\left|S\left(e_{i}\right)\right| \geqslant$ $4(m-2)+2+2 \geqslant 4 m-4$, which contradicts $(2)$. Thus we may assume that no edge in $M$ satisfies (ii') or (ii") of Claim 1. Moreover, if (ii) of Claim 1 holds for some $e_{i}$, then since $S\left(e_{i}\right) \cap S\left(e_{j}\right)=\emptyset$ for every $j \neq i$, each edge in $M \backslash\left\{e_{i}\right\}$ satisfies (i) of Claim 1 . Then $|S| \geqslant \sum_{i=1}^{m}\left|S\left(e_{i}\right)\right| \geqslant 4(m-1)+2 \geqslant 4 m-2$, which contradicts (2). Thus we may assume that no edge in $M$ satisfies (ii) of Claim 1.

If (iii) of Claim 1 does not hold for any $e_{i}$, then $|S| \geqslant \sum_{i=1}^{m}\left|S\left(e_{i}\right)\right| \geqslant 4 m$, which contradicts (2). Hence (iii) of Claim 1 holds for some $e_{i}$, and thus there exists a component of $G-K$ which contains a non-separating 2 -sided non-contractible cycle. Let $D_{1}, \ldots, D_{k}$ be such components of $G-K$. In the case $k \geqslant 3$, let $H_{1}, \ldots, H_{l}$ be the components of $G-\left(V\left(D_{1}\right) \cup \ldots \cup V\left(D_{k}\right)\right)$. Moreover, let $S_{i}=S \cap H_{i}$ and let $c_{i}$ be the number of components of $G-K$ which are contained in $H_{i}$. Assume that $c_{j} \leqslant\left|S_{j}\right|-1$ for some $j$. Then $G^{\prime}-\left(S-S_{j}\right)$ has at least $|S|+2-\left(c_{j}+1\right) \geqslant|S|+2-\left|S_{j}\right|=\left|S-S_{j}\right|+2$ components, which contradicts the minimality of $S$. Thus $c_{i} \geqslant\left|S_{i}\right|$ for every $i$. However, $c_{o}(G-K)=k+\sum_{i=1}^{l} c_{i} \geqslant k+\sum_{i=1}^{l}\left|S_{i}\right| \geqslant|S|+3$, a contradiction. Therefore we have $k \leqslant 2$.

Without loss of generality, we may assume that, for some integer $h \geqslant 1$, edges $e_{1}, \ldots, e_{h}$ satisfy (iii) of Claim 1 . For each $i=1, \ldots, h$, let $C_{i}$ be a non-separating 2-sided noncontractible cycle in $G\left[S\left(e_{i}\right) \cup V\left(e_{i}\right)\right]$ that qualifies $e_{i}$ for (iii) of Claim 1, and let $Q_{1}$ $\left(Q_{2}\right)$ be a non-separating 2-sided non-contractible cycle contained in $D_{1}\left(D_{2}\right)$. Then $C_{1}, \ldots, C_{h}, Q_{1}, Q_{2}$ are homotopic to each other. Let $A_{1}, \ldots, A_{h}$ be annuli obtained by cutting the torus or the Klein bottle along $C_{1}, \ldots, C_{h}$. Then there are at most two annuli 
which contain $D_{1}$ or $D_{2}$, and at most four cycles in $C_{1}, \ldots, C_{h}$ are the boundaries of such annuli. Consequently for every $j$ such that $C_{j}$ is not in the above four cycles, $S\left(e_{j}\right)$ can have a neighbor neither in $D_{1}$ nor in $D_{2}$. Hence at most four edges of $M$ satisfies (iii) of Claim 1. Therefore $|S| \geqslant \sum_{i=1}^{m}\left|S\left(e_{i}\right)\right| \geqslant 4(m-4)+3 \cdot 4=4 m-4$, which contradicts (2).

\section{Acknowledgments}

The authors would like to thank Atsuhiro Nakamoto for his valuable comments.

\section{References}

[1] R. E. L. Aldred and M. D. Plummer. Edge proximity and matching extension in planar triangulations. Australas. J. Combin., 29:215-224, 2004.

[2] R. E. L. Aldred and M. D. Plummer. Restricted matching extension in graphs of small genus. Discrete Math., 308:5907-5921, 2008.

[3] R. E. L. Aldred and M. D. Plummer. Distance-restricted matching extension in planar triangulations. Discrete Math., 310:2618-2636, 2010.

[4] R. E. L. Aldred and M. D. Plummer. Proximity thresholds for matching extension in planar and projective planar triangulations. J. Graph Theory, 67:38-46, 2011.

[5] R. E. L. Aldred and M. D. Plummer. Proximity thresholds for matching extension in the torus and Klein bottle. Discrete Applied Math., 160:985-991, 2012.

[6] R. Diestel. Graph Theory. Second edition. Graduate Texts in Mathematics, 173. Springer-Verlag, New York, 2000.

[7] K. Kawarabayashi, S. Negami, M. D. Plummer and Y. Suzuki. The 2-extendability of 5-connected graphs on surfaces with large representativity. J. Combin. Theory Ser. B, 101:206-213, 2011.

[8] I. Mizukai, S. Negami and Y. Suzuki. The 2-extendability of graphs on the projective plane, the torus and the Klein bottle. Graphs Combin., 26:549-557, 2010.

[9] M. D. Plummer. A theorem on matchings in the plane. Ann. Discrete Math., 41:347354, 1989.

[10] M. D. Plummer. Extending matchings in planar graphs IV. Discrete Math., 109:207219, 1992. 